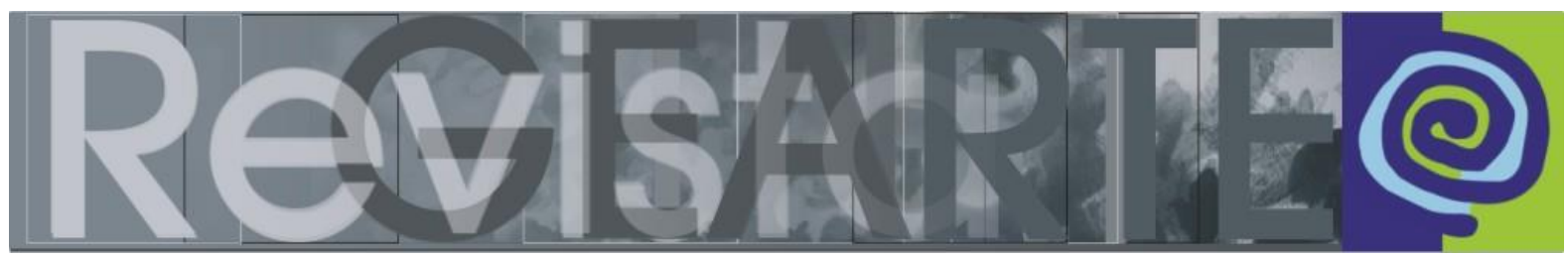

e-ISSN 2357-9854

\title{
La búsqueda del perfil docente en educación artística a través del mPortafolio
}

\author{
Noemi Peña Sanchez \\ (Universidad de La Laguna - ULL, San Cristóbal de La Laguna, \\ Santa Cruz de Tenerife, España)
}

\begin{abstract}
RESUMEN - La búsqueda del perfil docente en educación artística a través del mPortafolio Este artículo reflexiona sobre las ventajas del portafolio de aprendizaje a través del uso de aplicaciones móviles educativas y que genera lo que hoy denominamos como mPortafolio. Al mismo tiempo, pretendemos aprovechar la inevitable presencia de los dispositivos móviles en el aula superando el dilema sobre su uso, al tiempo que resignificar estas tecnologías digitales para dotarlas de valor educativo en la formación en Educación Artística. En el estudio presentado abordamos dos temas de interés. Por un lado, el papel del portafolio de aprendizaje como recurso para la reflexión individual y colaborativa del aprendizaje dentro y fuera del aula de Educación Artística. Por otro lado, investigar desde la práctica docente las posibilidades que brindan los dispositivos móviles ensayando formas participativas de aprendizaje que conviertan el aula en un espacio híbrido entre lo presencial, lo digital e interactivo.
\end{abstract}

PALABRAS CLAVE

mPortafolio. Reflexión como aprendizaje. Educación Artística. Educación superior.

ABSTRACT - Looking for a teaching philosophy in art education through mPortfolio - This article reflects on some advantages of the learning portfolio through the use of mobile educational applications which is known as mPortfolio. In addition, the inevitable presence of mobile devices in the classroom provides a great resource for our student's learning, overcoming the dilema over its use. We also need to redefine these digital technologies by giving them an educational value for Arts Education. This study addresses two particular issues. On the one hand, the role of the learning portfolio as a resource for individual and collaborative learning reflection, both inside and outside the Arts Education classroom. On the other hand, to investigate the possibilities of mobile devices from our teaching practice while experimenting with participatory forms of learning that turn the classroom into a hybrid space between classroom, digital and interactive.

\section{KEYWORDS}

mPortfolio. Learning reflection. Mobile aplication. Art education. Higher Education.

\section{Introducción}

Presentamos el mPortafolio como una de las propuestas formativas enmarcadas en el proyecto de innovación Centros digitales de aprendizaje: tiempo, participación y reflexión desarrollado en la Universidad de La Laguna. Como reto proponemos la innovación en metodologías de enseñanza y aprendizaje a través del uso de tecnologías de información y comunicación, en especial el uso de aplicaciones digitales en los dispositivos móviles. En esta experiencia utilizaremos la aplicación digital Seesaw class que ofrece la creación de aulas virtuales de apariencia similar a 


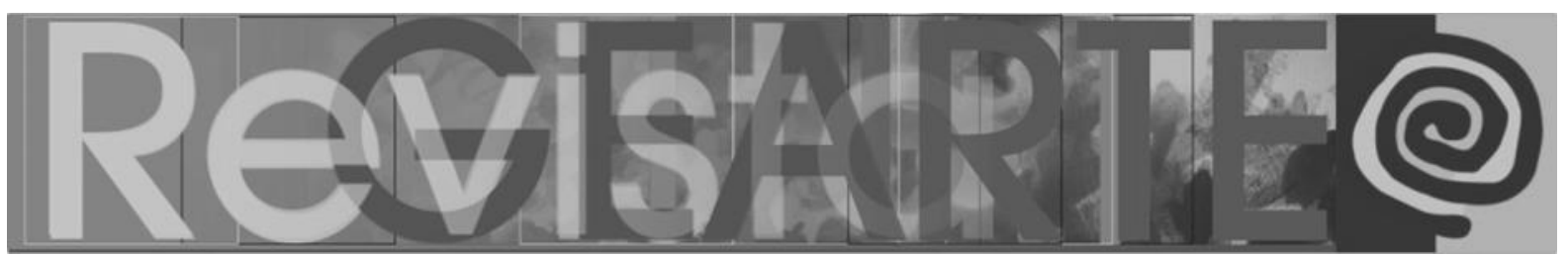

redes sociales con las que el estudiante generará su mPortafolio de aprendizaje recopilando evidencias y reflexionando sobre su proceso de aprendizaje.

Analizaremos también el valor del portafolio de aprendizaje desde las aportaciones de dos autores Barret (2012) y Hernández (2008) para entender las claves necesarias para plantear un mPortafolio en el aprendizaje de las artes como enseñanza. Propondremos también dos niveles para el desarrollo del mPortafolio en el que la reflexión juega un papel clave para alcanzar los objetivos propuestos.

\section{El portafolio para reflexionar sobre la formación docente en educación artística}

Para contextualizar y fundamentar el uso del portafolio es necesario comprender el papel que ocupa la formación en educación artística en las titulaciones universitarias de carácter oficial de la Universidad de La Laguna. Quisiera concretar que al definir educación artística nos estaríamos refiriendo a la denominación de educación artística desde las artes visuales. Actualmente nuestra formación didáctica se imparte en tres titulaciones de grado y dos másteres. Actualmente los planes de estudio de los grados de Educación Infantil y Primaria cuentan únicamente con una asignatura de carácter obligatorio y de duración cuatrimestral impartida en $3^{\circ}$ y $4^{\circ}$ ำ curso respectivamente en cada una de estas titulaciones. Esta formación en educación artística de los futuros maestros y maestras resulta sin lugar a dudas insuficiente para abordar dos objetivos fundamentales. En primer lugar, necesitan individualmente superar los miedos, barreras y dificultades de las prácticas artísticas y por otro lado, ser capaz de desarrollar experiencias artísticas significativas adecuadas a cada contexto en las etapas correspondientes, bien sea Educación Infantil y Primaria.

En la titulación de Grado de Bellas Artes existe una asignatura de carácter optativo para aquellos estudiantes que en sus últimos cursos universitarios quieran iniciarse en la educación artística y conocer así las posibilidades que el arte ofrece desde una perspectiva educadora y comunitaria en contextos educativos no formales. En este caso, los estudiantes poseen las habilidades y conocimientos de lenguajes 


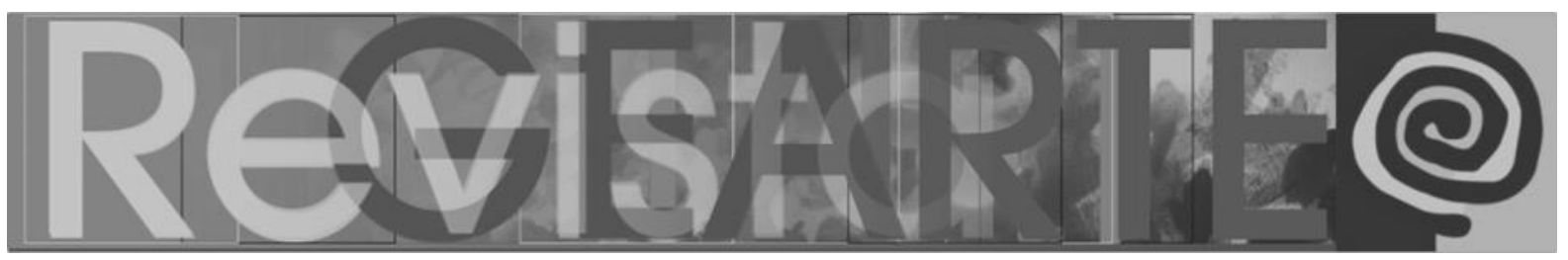

artísticos, sin embargo, necesitan desarrollar esas habilidades comunicativas y una formación didáctica para enseñar a través de las artes. Es llamativa la necesidad de resignificar el concepto de educación artística y superar estereotipos en torno al papel del educador/a.

Respecto a aquellas titulaciones posteriores al grado, quisiera destacar especialmente el Master habilitante en Formación del profesorado de Educación Secundaria Obligatoria y Bachillerato en la especialidad de Dibujo, Diseño y Artes plásticas de duración anual. Esta titulación académica es la que mayor presencia cuenta en la formación en educación artística. El alumnado, aunque proviene de diferentes titulaciones como Bellas Artes, Diseño y Arquitectura, también demanda una formación didáctica necesaria para comprender no solo qué enseñar, sino también cómo enseñarlo adecuándolo al contexto formal de la etapa en la que nos encontremos.

Esta formación didáctica acotada en asignaturas de intensa duración no propicia espacio alguno de reflexión para ese futuro educador, el cual le permitiría entender la tarea docente en toda su complejidad. De modo, que una de las propuestas que les planteamos es la creación de un portafolio de aprendizaje que nos permita maximizar el tiempo del aula para reflexionar sobre todas aquellas cuestiones que se suceden en el aula y de las que podemos extraer cuestiones de interés para su formación didáctica.

Hernández (2008) subraya la importancia del portafolio para mejorar la calidad educativa al desplazar el foco de interés hacia el proceso de aprendizaje en lugar de centrarlo únicamente en los resultados. El portafolio que nos interesa propicia una situación en la que el estudiante debe entender e interiorizar ese aprendizaje para incorporarlo progresivamente a su rol docente. Paulson y Meyer (1991) lo describen así: "un portafolio es un laboratorio donde los estudiantes construyen significados a partir de la experiencia acumulada"(citado por HERNÁNDEZ, 2008, p.61). 


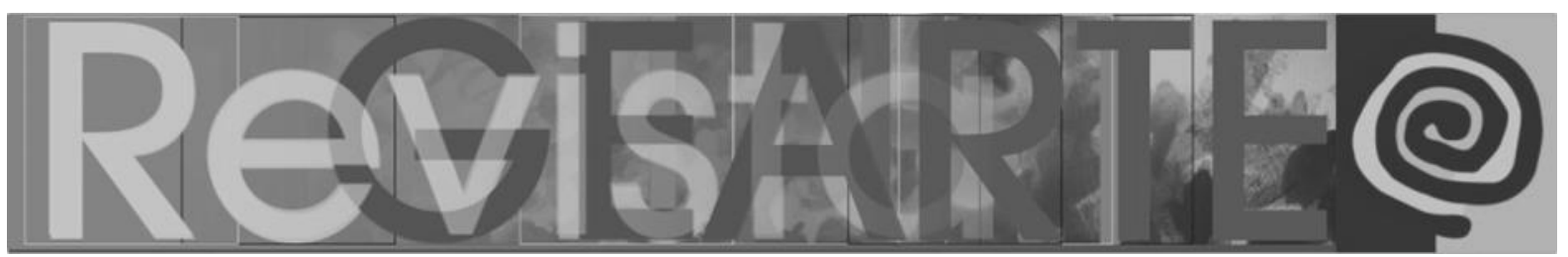

\section{Aplicaciones móviles como espacios de participación en el aprendizaje}

En la sociedad actual en la que vivimos hemos aprendido a manejar y comunicarnos a través de nuestros móviles o celulares en nuestro día a día y casi nos parece inconcebible un modo de comunicación que prescinda de esta tecnología. Para la generación del Milenio y de esos nativos digitales que nacieron ya rodeados de dispositivos móviles, éstos son además una parte identitaria con la que configuran su personalidad a través de las diferentes redes sociales y con las que son capaces de crear contenido y comunicarse a velocidad asombrosa. Según Scolari (2016) los jóvenes llevan más de una década participando activamente en culturas colaborativas, entendidas éstas como comunidades virtuales con las que conectan y participan como prosumidores (consumidores y productores). De hecho, Jenkins (citado por Scholari, 2016) identifica cuatro modos de participación en estas culturas colaborativas que consisten en asociación, expresión, resolución colaborativa de problemas y circulación. Cada una de ellas define claramente esa denominación de prosumidor, participando tanto como consumidor de información como productor, o más bien creador, publicando contenido y compartiéndolo en esa red con otros usuarios.

Si pensamos en la transferencia de esta realidad a nuestros contextos educativos somos conscientes del camino que aún debemos recorrer para adaptarnos a esa otra realidad. Es por eso que debemos ser conscientes de esa hipervisualidad conectada de la que forman parte nuestros estudiantes hoy $y$, sin duda, las generaciones venideras.

Sin duda, nuestro posicionamiento pretende apropiarse de esa vivencia intensa y utilizar los dispositivos móviles para aprovecharnos de las funcionalidades que puede aportar para nuestro aprendizaje. Para Mc Caffrey (2011) se trata de superar esa idea disruptiva que supone el uso de la tecnología móvil en el aula y preguntarnos en realidad cómo debería ser ese un aprendizaje que integre y tome las ventajas para reformular la educación. La autora apunta cómo esta tecnología móvil nos ayuda a dar respuesta a dos interrogantes en la educación actual "el deseo de los estudiantes para aprender diferente y la necesidad de los estudiantes por aprender diferente" 


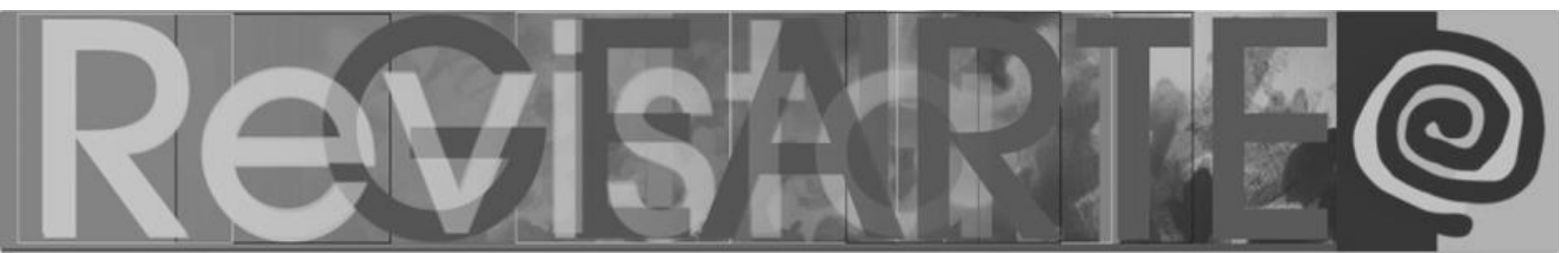

(02/08/11). El deseo proporciona esa motivación por el uso de los móviles, al tiempo que proporciona espacios educativos virtuales con los que necesitan comunicarse al igual que sucede en las redes sociales.

Somos conscientes de que la educación actual en nuestro sistema universitario cuenta ya con plataformas virtuales de aprendizaje como Moodle con las que virtualmente podemos trabajar y diseñar actividades. Sin embargo, esta plataforma es administrada únicamente por el o la profesora, quien debe encargarse de estructurar y diseñar el contenido, así como las actividades de aprendizaje. El estudiante participa únicamente a través de estas herramientas con poco margen para interactuar con otros compañeros/as. Si comparamos este tipo de plataformas y las comunidades virtuales existentes ponen en evidencia esa brecha tecnológica que existe.

Actualmente podemos encontrarnos diferentes aplicaciones con una función educativa para interactuar en el aula. La aplicación móvil utilizada es Seesaw class con la que podemos crear clases como si se tratase de una comunidad virtual e invitando a los estudiantes a que formen parte de manera protegida y segura. Este espacio virtual ofrece al estudiante un espacio de participación como prosumidor ya que puede crear sus contenidos, al tiempo que consume también las que otros estudiantes y profesor/a generan. En la actualidad Seesaw class es una aplicación de carácter gratuita que permite la descarga tanto en tabletas digitales, móviles o incluso acceder desde un ordenador a través de su página web. Esta aplicación ha sido diseñada para el sistema educativo americano desde las etapas de Kindergarten a grado 12 (K-12), sin embargo podemos crear otro tipo de aulas adaptando sus ajustes a las necesidades del contexto de la educación universitaria.

Podemos destacar algunas de las ventajas concretas que mencionábamos. En primer lugar, la aplicación resulta dinámica, de fácil acceso y con una configuración similar a la de una red social, aunque en este caso cerrada al grupo clase. Este formato dota a cada estudiante de un perfil, similar al que configuran en otras redes y al que todos ellos pueden tener acceso. Otra de las diferencias que encontramos frente a otras plataformas educativas es la de posibilitar ese perfil de prosumidor que 


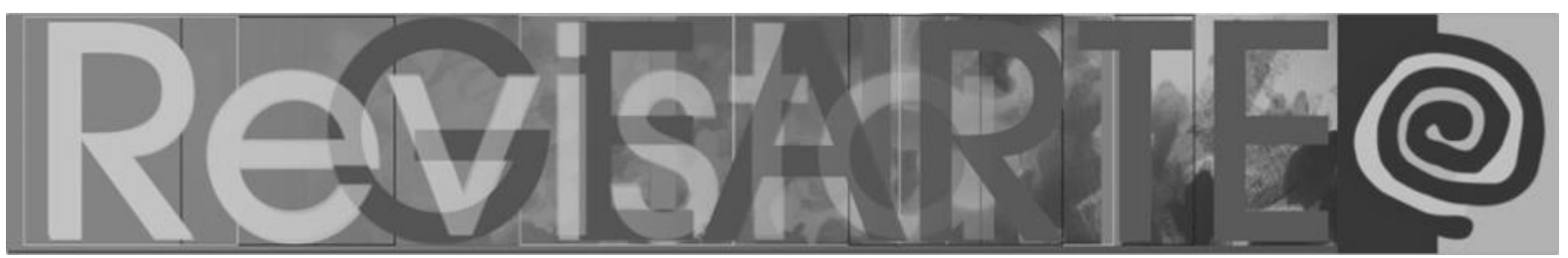

encontramos en otras comunidades virtuales. Al ser parte activa pueden participar desde el aula en la comunidad virtual educativa publicando sus entradas en formato de nota, imágenes, fotografías, vídeo y hasta grabación de voz. Sin duda, uno de sus potenciales es la inmediatez en la visibilización de contenidos dando voz a todos y cada uno de los estudiantes en el aula y haciéndoles participar activamente de su aprendizaje. Aguirre, Olaiz, Marcellán, Arriaga y Vidador (2010) subrayan también la importancia de situar a los estudiantes en productores, en lugar de situarlos únicamente en consumidores.

Si observamos las actividades culturales más usuales en los entornos juveniles, inmediatamente percibimos que la tecnología por un lado y las industrias de la cultura y el ocio por otro, otorgan cada vez más protagonismo a la faceta creadora del hasta hace poco mero usuario consumidor de la cultura visual (AGUIRRE et.ali, 2010, p.1).

Seesaw class nos proporciona así experiencias de aprendizaje que sitúan a los estudiantes en ese perfil de creador estrechamente vinculado con ese quehacer cotidiano que mantienen durante su tiempo de ocio. En el gráfico siguiente queremos destacar tres ventajas sobre el uso de esta aplicación digital en el aula.

\section{Figura 1 - Ventajas de la aplicación Seesaw Class}
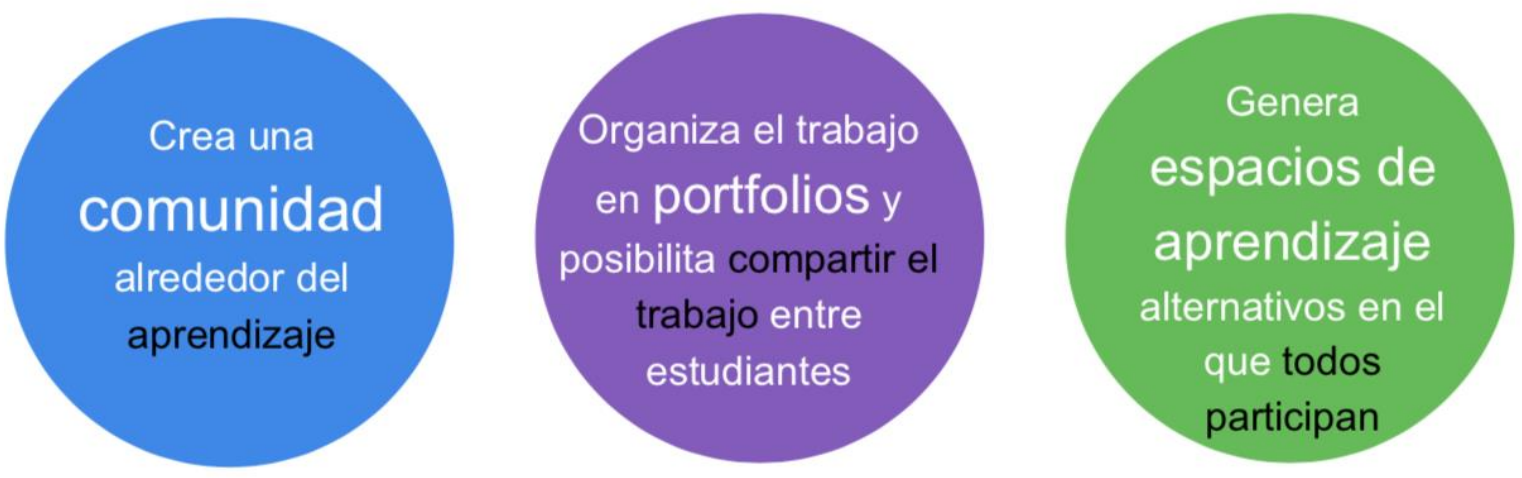

Fuente: Elaboración de la autora

\section{Del ePortafolio al mPortafolio}

Butler (2006) describe el portafolio como una recopilación de evidencias que son acumuladas y organizadas a lo largo de un periodo de tiempo con el propósito de 


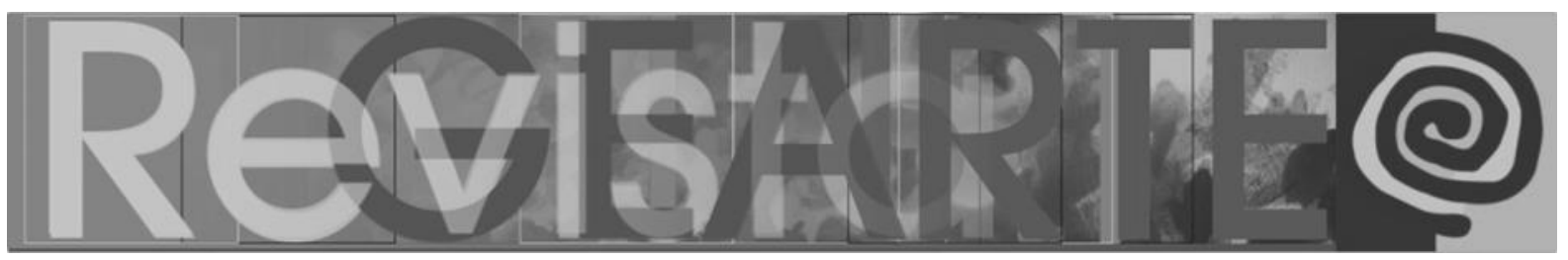

demostrar unas habilidades sobre un aprendizaje. Barret (s.f.) al definir el portafolio digital o ePortfolio hace referencia también a esa idea de colección de evidencias, aunque en un formato digital y subrayando la reflexión como acción clave dentro de ese proceso de aprendizaje. Las evidencias entendidas éstas como productos en formato de texto, imagen, audio, vídeo o combinación de varios formatos, requieren de una acción meditada para ser significadas. La elección de aquellas evidencias que forman parte del portafolio implica también una reflexión que necesita ser argumentada para conocer ese aprendizaje. Hernández (2008) afirma que desde ese posicionamiento el valor del portafolio digital no debe residir en la mera recopilación de evidencias, sino en los argumentos que el estudiante aporte sobre ellos y que sean reflejo de haber alcanzado unos resultados del aprendizaje. En la experiencia innovadora descrita, plantean el uso del ePortafolio como una forma alternativa de enseñar no solo centrada en el qué, referida ésta a la colección de evidencias, sino también en el cómo se han producido esos aprendizajes y lo que significan en el proceso vital de formación del estudiante.

Este planteamiento del ePortafolio es igualmente válido para el portafolio móvil o mPortafolio, la diferencia estriba en dos cuestiones: el formato y forma de almacenamiento de todas esas evidencias de aprendizaje y la visibilidad de estos artefactos para otros estudiantes. Las evidencias del mPortafolio van a tener un formato específico por la propia configuración que la aplicación móvil ofrece. Esas evidencias pueden adoptar diferentes formatos como nota de texto, grabación de voz, vídeo, imagen e incluso la combinación de estos recursos. Todas ellas deben tener en común la recogida de los procesos de aprendizaje significativos durante cada sesión de clase. Otro aspecto singular es la visibilización de esas evidencias, entendidas éstas como aportaciones al mPortafolio. El hecho de que éstas puedan ser accesibles al resto de estudiantes es una forma de motivación intrínseca que estimula al estudiante en un espacio y tiempo más allá del aula. La publicación de estas aportaciones se hace en un espacio virtual compartido, seguro y en el que los usuarios de esa red pueden acceder en tiempo real a las aportaciones elaboradas por el resto de los estudiantes que en ella participan. 


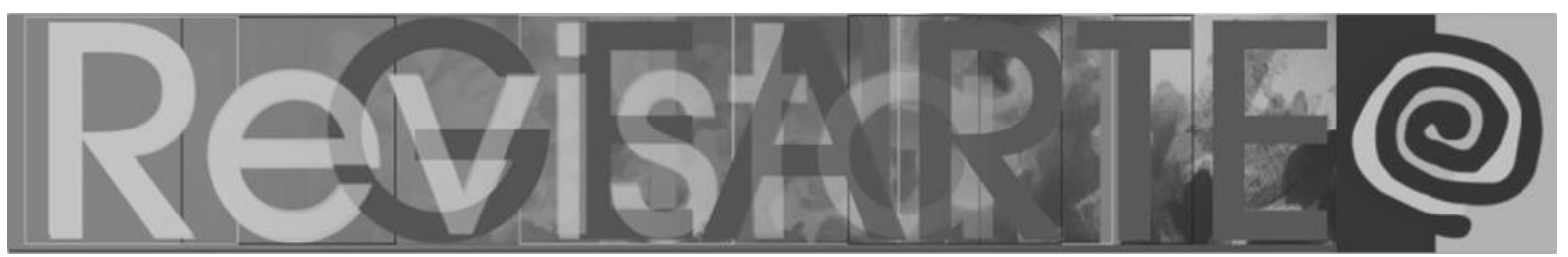

Uno de los aspectos que debe ser prioritario a la hora de plantear la creación del portafolio, ePortafolio y mPortafolio a nuestros estudiantes es el hecho de definir los objetivos que pretendemos alcanzar. Hernández (2008) nos aconseja que debemos ser cuidadosos y no enfocarlo como tarea hacia la evaluación ya que el estudiante puede entenderla como una obligación por su calificación, cuando en realidad buscamos una implicación más vivencial y reflexiva hacia el aprendizaje.

\section{Niveles de desarrollo en el planteamiento de un mPortafolio en la formación en educación artística}

El mPortafolio que hemos planteado está dirigido a los estudiantes en las asignaturas de educación artística de las titulaciones de Bellas Artes y del Máster de Formación del Profesorado en Secundaria y Bachillerato en la especialidad de Dibujo, Diseño y Artes Plásticas. Como inicialmente comentábamos estas materias requieren de procesos de aprendizaje reflexivos que unidos al escaso tiempo de las asignaturas nos hace plantearnos una búsqueda de otros recursos que permitan maximizar el tiempo del aula y posibilitar al estudiante extraer aprendizajes que pueda llevar a su práctica docente.

Uno de nuestros objetivos para ese mPortafolio es que el estudiante sea capaz de apropiarse de aprendizajes, de modos de enseñar y que entienda cómo puede transferirlos a otros contextos educativos en su futura práctica docente. Promover el hábito de esa reflexión en torno al aprendizaje y retroalimentarse de los comentarios y aportaciones de otros para finalmente ir definiendo su perfil docente.

Para plantear el desarrollo del mPortafolio en nuestras asignaturas nos hemos basado en los tres niveles propuestos por Barret (2012) para el desarrollo de un portafolio en los que secuencia a su vez acciones progresivamente más complejas que parten de la simple recopilación hacia la reflexión hasta llegar a generar un aprendizaje profundo y sincero. En un primer nivel, la autora se refiere a la colección de evidencias o artefactos que va recopilando durante su aprendizaje, en las que el estudiante dispone de sistemas de almacenamiento variados que deben adecuarse al 


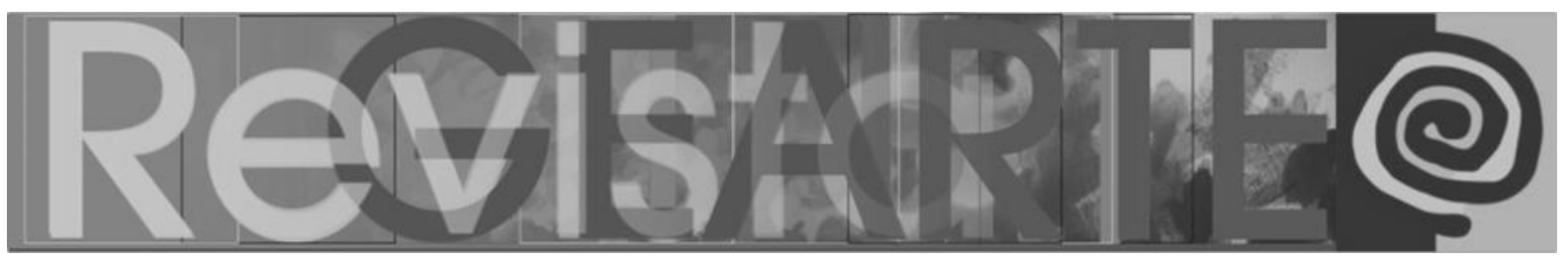

formato de la información. El segundo nivel está centrado en la narración de esas evidencias y todo aquello que las rodea, en ese ejercicio de reflexión es fundamental afrontarlo desde la vivencia del aprendizaje. Para Harris (2006) la reflexión y en especial la reflexión crítica nos obliga a conectarnos con nosotros mismos, a ser honestos y la profundizar en las ideas hasta llegar a un aprendizaje profundo de las cosas, es lo que Hernández (2008) define como "encontrar sus propias voces". Barret (2012) coincide en la importancia de trabajar la reflexión, proporcionando al estudiante una especie de andamiaje, para que progresivamente sepa orientar y realizar reflexiones con las que llegue a encontrar su voz. La retroalimentación del profesor/a, la exploración de estrategias en torno a la reflexión son algunas de las recomendaciones que Barret (2012) sugiere para que estas narraciones no queden en la mera superficie. Finalmente en un tercer nivel hablaríamos de realizar una selección de esas evidencias o artefactos de valor para la presentación final de ese portafolio. Sin duda el diseño y presentación de ese portafolio debiera responder a los objetivos planteados inicialmente.

Redefiniendo el modelo propuesto por Barret (2012), nuestro mPortafolio se desarrollaría en dos niveles de desarrollo. Un primer nivel de colección y reflexión de evidencias que dan respuesta a ¿qué aprendizajes me llevo? Y ¿Cómo se producen? En un segundo nivel estaría presente esa acción reflexiva y podemos decir sumativa que reflexione desde otro posición temporal a partir de una observación retrospectiva y concretando ¿Qué elementos clave aparecen en las aportaciones? ¿Cómo éstas pueden definir mi perfil docente?

\section{1ํNivel colección-reflexión ¿Qué aprendizajes me llevo?}

Una de las necesidades de adecuar ese planteamiento es la configuración y versatilidad que ofrecen las aplicaciones móviles, que como ya mencionamos se trata de Seesaw class. Esta aplicación cuenta con una herramienta muy apropiada para el desarrollo de portafolios, configurada como diario o journal, con la cual se van creando de manera sencilla e inmediata diferentes entradas/posts con las que se van generando un mPortafolio. De este modo, cada estudiante va progresivamente 


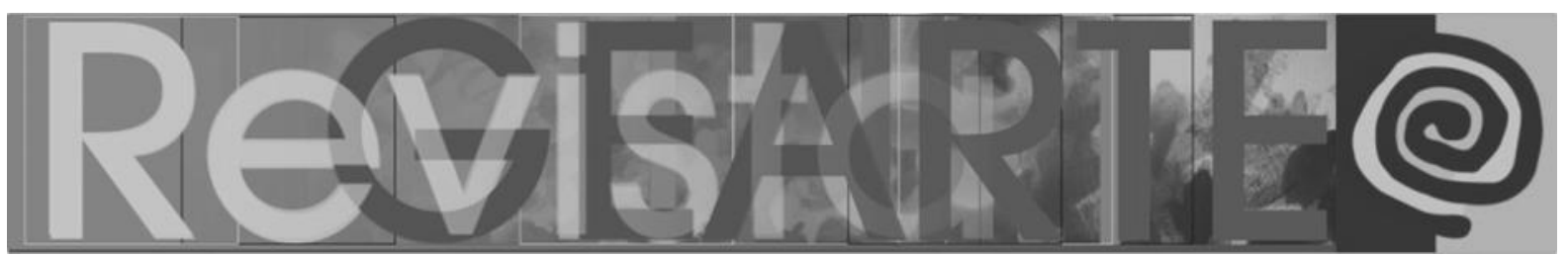

trabajando en su portafolio de aprendizaje, lo que nos permite ir proporcionando recursos y estrategias para profundizar en esa reflexión. Durante este proceso correspondiente a ese primer nivel de implementación del mPortafolio implica un tipo de participación diferente al que define Barret, puesto que se trata de un espacio compartido, similar al de una red social. Las reflexiones aportadas son visualizadas en tiempo real por todos los miembros de ese grupo clase, lo que implica una mayor responsabilidad al estudiante a la hora de publicar sus entradas. Al mismo tiempo, éstas pueden servir también de referencia para reconsiderar el planteamiento de cada reflexión individual realizada. Es también enriquecedor para el estudiante recibir comentarios o "me gusta" en las diferentes entradas, de modo que reciba una retroalimentación directa por parte de todos los integrantes de la red del grupo clase.

Una de las ventajas de este diario es la posibilidad combinatoria de lenguajes que podemos utilizar. Si bien es cierto que la herramienta nota de texto es la más utilizada por nuestros estudiantes, también pueden incluirse dibujos, fotografías o vídeos, así como la creación de comentarios de voz. En realidad, una imagen puede ser reveladora para el estudiante no solo por lo que lo que ésta describa, sino por el contexto de la experiencia educativa que allí sucede. Además, cada herramienta es combinable con cualquier otra, lo que permite al estudiante elegir la mejor forma de crear un post de acuerdo con el contenidos que se trabajen. Aunque en nuestro planteamiento, la creación de entradas para ese mPortafolio se realiza al finalizar cada sesión, su cantidad debe estar abierta a recopilar y crear nuevas evidencias en función de los intereses encontrados durante ese aprendizaje.

\section{2ํNivel reflexión sumativa ¿Cómo describiría mi perfil docente?}

El paso final implica lo que Barret (2012) denomina muestra o presentación del producto final (showcase) que muestra todo ese proceso de trabajo del mPortafolio. Para su elaboración la autora habla de la selección de aquellos artefactos o evidencias que sean representativos del proceso de aprendizaje y que den evidencias del logro de esos objetivos propuestos inicialmente. Dicha selección implica una revisión de esos artefactos, pero desde una perspectiva diferente. Esa lectura se realiza en un 


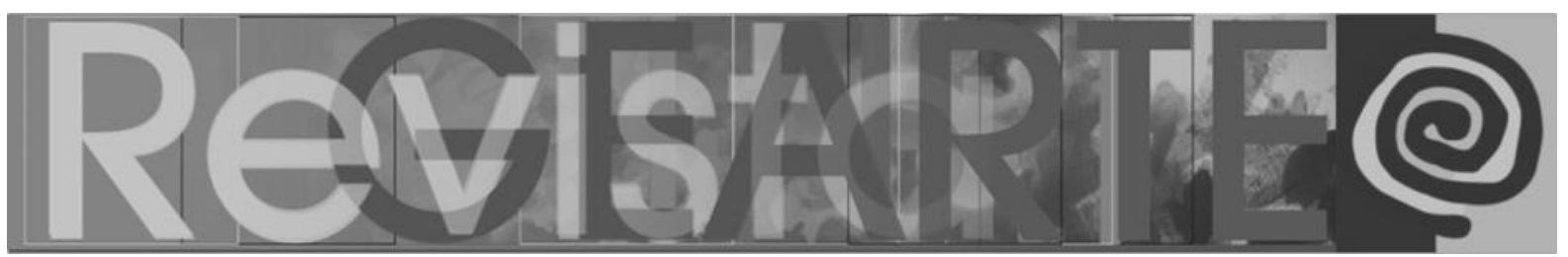

espacio temporal diferente al que se fueron realizando cada una de esas aportaciones o entradas durante el desarrollo del mPortafolio. Nos referimos a que esta reflexión exige una mirada retrospectiva como visión global de todo ese aprendizaje dando lugar a nuevas reflexiones que conectan y establecen relaciones antes no detectadas. En nuestro m-Portafolio pensamos que es fundamental realizar esta reflexión sumativa porque el estudiante toma conciencia de los avances que pueden producirse durante un periodo de tiempo y llegar a ese aprendizaje profundo en el que dará respuesta al interrogante inicial planteado para el mPortafolio.

\section{Conclusiones}

Una de las cuestiones que debemos apuntar es la distancia que aún existe entre los avances tecnológicos actuales y los recursos que manejamos dentro de las instituciones educativas. Sin duda, nuestro tiempo de ocio se ha modificado en los últimos años generando nuevos hábitos que antes ni podíamos imaginarnos. En nuestras aulas, aún nos es difícil trasladar esas nuevas maneras de comunicarnos y entretenernos a través de aplicaciones, manejando otros nuevos lenguajes simbólicos y hasta generando perfiles que nos representen a través de redes sociales. Los jóvenes como nativos digitales son los que apropian eficazmente de este tipo de hábitos más naturalmente y es necesario conocerlos para saber de que manera pueden ser transferibles a nuestras aulas. Scolari (2016) nos habla de un alfabetismo transmedia que complementa esa visión crítica que enseñamos acerca de los medios de comunicación.

\footnotetext{
Es un enfoque complementario que se desplaza de la enseñanza tradicional (enseñar habilidades críticas de comunicación en la escuela) hacia el aprendizaje informal (entender cómo las nuevas generaciones están haciendo cosas con los medios fuera de la escuela). (SCOLARI, 2006, p. 21).
}

De hecho, la creación de un mPortafolio a través de la aplicación móvil Seesaw class, supone ensayar con esos espacios híbridos que combinan lo informal con lo formal, lo presencial con lo digital e interactivo. Sin duda, durante el desarrollo del portafolio móvil de aprendizaje hemos experimentado posibilidades que desconocíamos y con las que resignificar prácticas para ampliar nuestras 


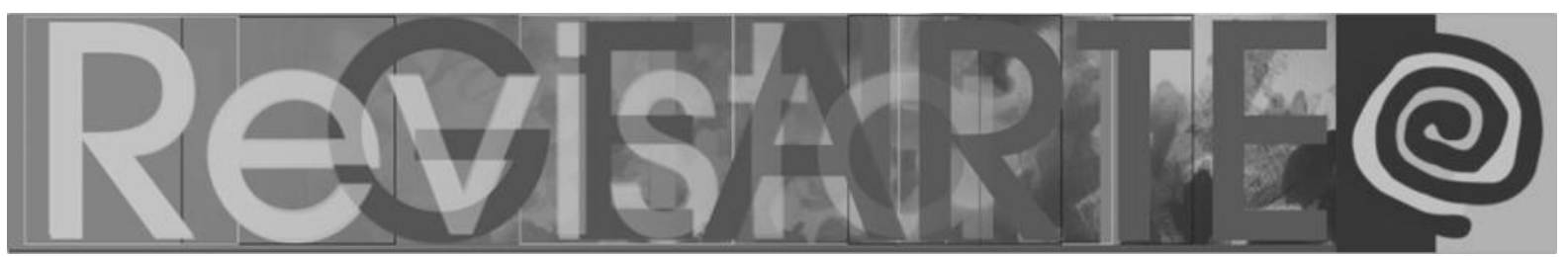

experiencias. Aprendemos así a interactuar de forma diferente con nuestros estudiantes e incluso entre ellos mismos. Precisamente, una de las fortalezas es la proximidad y relación que podemos establecer con cada uno de ellos en ese espacio individual y compartido de su mPortafolio. No cabe duda que debemos seguir innovando y ensayando propuestas que conecten las realidades del aula con las necesidades que nuestros estudiantes demandan para su futuro aunando así lo formal e informal.

\section{Referências}

AGUIRRE, I., OLAIZ, I., MARCELLÁN, I., ARRIAGA, A., y VIDADOR, M. (2010). Estudio sobre jóvenes productores de cultura visual: un estado de la cuestión. En Congreso lberoamericano de Educación. METAS 2021. 1-26.

BARRET, H. Electronic Portfolios and Digital Storytelling for lifelong and life wide learning. Disponible en: <https://electronicportfolios.org>, Acceso en 23 feb. 2019.

BARRET, H. M-portfolios. Disponible en <https://sites.google.com/site/mportfolios/course-lessons>, Acceso en 26 de feb.2019

BARRET, H., y RICHTER, J. Reflection 4Learning. Disponible en <https://sites.google. com/site/reflection4learning/Homes, Acceso en 26 feb. 2019.

BUTLER, P. Review of the Literature on Portfolios and Eportfolios. 2016. Disponible en <https://eduforge.org/docman/view.php/176/1111/ePortfolio\%20Project\%20Research\%20Report.pdf > Acceso en 24 feb. 2019.

HARRIS, D. The Power and Practice on Reflection. Professional Development: A cornestone Strategy for Change.vol.3, no 1, feb. 2006.

HERNÁNDEZ, F. El portafolio y el aprendizaje para la comprensión en secundaria. Investigación en la escuela, Sevilla, Universidad de Sevilla, 64, pp.59-71, 2008.

JENKINS, H., FORD, S, GREEN, J. Cultura transmedia. La creación de contenido y valor en una cultura en red. Barcelona; Gedisa, 2015.

JIMÉNEZ, L., AGUIRRE, I., y PIMENTEL, L.G. Educación artística, cultura y ciudadanía. O.E.I., 2011.

MC CAFREY, M. (2011). Why Mobiles is a Must. Disponible en: <https://thejournal.com/articles/2011 /02/08/why-mobile-is-a-must.aspx>, Acceso en 23 feb.2019.

PLATT, S. (2018). Nothing Changes if Nothing Changes: \#firstdayfirstimage. Exposure magazzine. Disponible en: https://medium.com/exposure-magazine/nothing-changes-if-nothing-changes$9362165 \mathrm{~d} 5 \mathrm{bca}$

SANCHEZ, Noemi Peña. Espacios digitales en el aula: Iniciativas participativas desde la educación artística. VIII Jornadas de Innovación Educativa. Tenerife, Universidad de La Laguna (en prensa).

SANCHO, J., y HERNÁNDEZ, F. La profesión docente en la era del exceso de información y la falta de sentido. RED. Revista de Educación a Distancia. 56, 2-23, 2018.

SCOLARI, C. Estrategias de aprendizaje informal y competencias mediáticas en la nueva ecología de la comunicación. Telos: Cuadernos de Investigación e Innovación, 103, 12-23, 2016.

SEESAW. The oficial website. Disponible en < https://web.seesaw.me>, Acceso en 23 feb. 2019. 


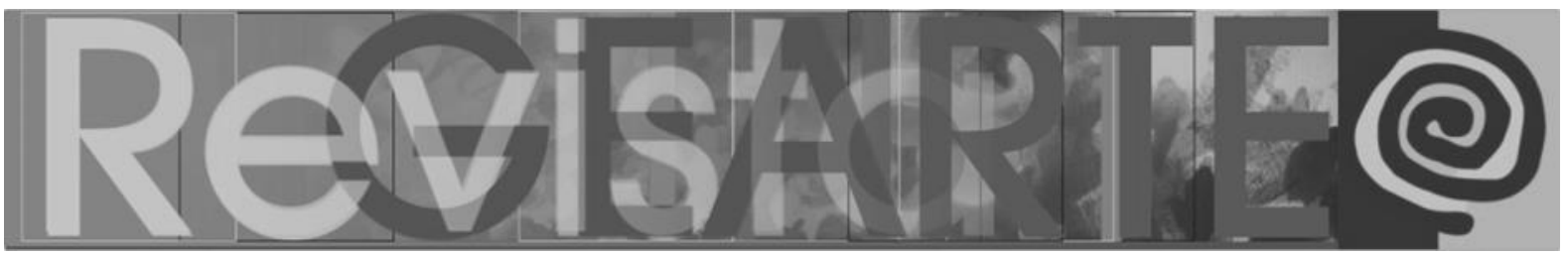

\section{Noemi Peña Sanchez}

Doctora en Bellas Artes por la Universidad Complutense de Madrid. Licenciada en Bellas Artes con la especialidad de Artes de la Imagen y Maestra en Educación Infantil. Actualmente profesora en el departamento de Bellas Artes, área de Didáctica de la Expresión Plástica impartiendo docencia en grados de Educación Primaria, Bellas Artes y Máster de profesorado en Educación Secundaria y Bachillerato. Coordinadora del área de Didáctica de la Expresión Plástica en la Universidad de La Laguna.

E-mail: npenasan@ull.edu.es

Currículo: https://www.ull.es/apps/guias/guias/view_teacher/118486/502/

Recebido em 28 de fevereiro de 2019 Aceito em 10 de abril de 2019 